\title{
O impacto da redução intensiva do c-LDL na morbimortalidade dos utentes com diabetes: revisão baseada na evidência
}

Carla Longras, Luís Filipe Cavadas, Cátia Cordeiro

\section{RESUMO}

Objetivo: Determinar o impacto da redução intensiva do c-LDL com estatina na morbimortalidade nos doentes com diabetes. Métodos: Foi realizada uma pesquisa de artigos em inglês, publicados entre 1 de janeiro de 2008 e 19 de julho de 2018 , com os termos: Hydroxymethylglutaryl-CoA Reductase Inhibitors AND diabetes mellitus AND low density lipoprotein AND morbidity e Hydroxymethylglutaryl-CoA Reductase Inhibitors AND diabetes mellitus AND low density lipoprotein AND mortality. Para atribuição dos níveis de evidência e forças de recomendação foi utilizada a escala SORT, da American Academy of Family Physicians (AAFP).

Resultados: Dos 174 artigos identificados, três foram selecionados: uma meta-análise e dois ensaios clínicos. A meta-análise incluiu 14 estudos e demonstrou o benefício da terapêutica com estatina na redução da incidência de eventos vasculares major em cerca de $1 / 5$ por $\mathrm{mmol} / \mathrm{L}$ de redução do c-LDL. No ensaio clínico prospetivo a redução de eventos foi maior no grupo que atingia valores de c-LDL menores; no entanto, sem diferença na mortalidade por todas as causas. No ensaio clínico retrospetivo, o grupo a tomar estatina verificou uma redução do risco cardiovascular e mortalidade.

Conclusões: Várias foram as limitações encontradas nos estudos analisados, nomeadamente por população heterogénea, follow-up de curta duração e limitações na qualidade dos estudos. Parece haver benefício na toma de estatina para obter valores baixos de c-LDL na redução da morbilidade, associada a ocorrência de eventos cardiovasculares, e na mortalidade (força de recomendação B). Contudo, existe evidência limitada sobre os valores alvo de c-LDL recomendados.

Palavras-chave: Estatinas; Diabetes mellitus; Lipoproteína de baixa densidade; Morbilidade; Mortalidade.

\section{INTRODUÇÃO}

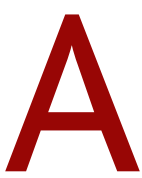
s pessoas com diabetes mellitus (DM) têm uma prevalência aumentada de alterações do metabolismo lipídico, contribuindo para o alto risco de desenvolverem doença cardiovascular (CV). ${ }^{1} \mathrm{O}$ benefício da redução de colesterol LDL (c-LDL) na redução do risco CV tem sido estudado neste grupo de pessoas. ${ }^{2}$

Segundo as normas da Direção-Geral da Saúde (DGS) em Portugal, é recomendado para pessoas com

*USF Lagoa, ULSM.
DM tipo 2 ou tipo 1, consideradas com risco cardiovascular alto (SCORE $\geq 5 \% \mathrm{e}<10 \%$ ), o controlo estrito do valor de c-LDL com valores inferiores a $100 \mathrm{mg} / \mathrm{dL}$. Se apresentam muito alto risco (SCORE $\geq 10 \%$ ), ou seja, se são pessoas com diabetes com um ou mais fatores de risco CV e/ou lesão dos órgãos-alvo, é recomendado o controlo estrito do valor de c-LDL para inferior a $70 \mathrm{mg} / \mathrm{dL} \cdot{ }^{3-4}$ Estas normas estão de acordo com as orientações de 2016 para a abordagem da dislipidemia da $E u$ ropean Society of Cardiology e da European Atherosclerosis Society (ESC/EAS), que recomendam no grupo de muito alto risco um valor de c-LDL $<70 \mathrm{mg} / \mathrm{dL}$, ou re- 
dução de pelo menos $50 \%$ se valor inicial estiver entre 70 e $135 \mathrm{mg} / \mathrm{dL}$, e no de alto risco um valor $<100 \mathrm{mg} / \mathrm{dL}$ ou redução em pelo menos $50 \%$ se valor inicial entre 100 e $200 \mathrm{mg} / \mathrm{dL} .^{5}$

Segundo as orientações, de 2013, da American College of Cardiology/American Heart Association (ACC/ (AHA) é recomendado o início de terapêutica com estatina com intensidade moderada em pessoas com diabetes com 40-75 anos e c-LDL entre 70 e 189mg/dL sem doença CV aterosclerótica, sendo que recomendam considerar a terapêutica com estatina de alta intensidade se c-LDL $\geq 190 \mathrm{mg} / \mathrm{dl}$ ou doença CV aterosclerótica clínica, exceto se idade superior a 75 anos, no qual se recomenda estatina de intensidade moderada. ${ }^{6}$

De acordo com as recomendações da American Diabetes Association (ADA), de 2018, pessoas com diabetes e doença $\mathrm{CV}$ possuem elevado risco e recomenda-se, portanto, a instituição da terapêutica com estatina de alta intensidade, tal como nas pessoas com diabetes com 40-75 anos com outros fatores de risco CV. Em pessoas com diabetes com menos de 40 anos de idade com fatores de risco para doença $\mathrm{CV}$ aterosclerótica ou pessoas com diabetes com 40-75 anos ou mais de 75 anos sem doença CV aterosclerótica e sem fatores de risco, para além das mudanças no estilo de vida, deve ser instituída terapêutica com estatina de moderada intensidade. $O$ ajuste da terapêutica com estatina deve ser baseado na resposta apresentada pelo doente à mesma (efeitos adversos, tolerabilidade, níveis do c-LDL ou percentagem de redução do c-LDL) até à dose máxima tolerada. $^{7}$

As estatinas são o fármaco de eleição para a redução de c-LDL e cardioproteção. Porém, deverão ser considerados os riscos da terapêutica intensiva. ${ }^{1-2}$ Esta revisão tem como objetivo determinar, à luz da evidência atual, o impacto da redução intensiva do c- LDL com estatina na morbilidade e mortalidade na população com diabetes.

\section{MÉTODOS}

Foi realizada uma pesquisa nas bases de dados PubMed, Guidelines Finder, National Guideline Clearinghouse, Canadian Medical Association Infobase, The Cochrane Library, DARE e Bandolier de revisões baseadas na evidência, normas de orientação clínica, meta-análises, revisões sistemáticas e ensaios clínicos alea- torizados, publicados desde 1 de janeiro de 2008 até 19 de julho de 2018, na língua inglesa, utilizando os termos MeSH: Hydroxymethylglutaryl-CoA Reductase Inhibitors AND diabetes mellitus AND low-density lipoprotein AND morbidity e Hydroxymethylglutaryl-CoA Reductase Inhibitors AND diabetes mellitus AND low-density lipoprotein AND mortality.

Os critérios utilizados para a inclusão dos artigos nesta revisão foram:

- População: indivíduos adultos com diabetes

- Intervenção: utilização de estatinas para atingir valores de LDL baixos $(<70$ ou $<100 \mathrm{mg} / \mathrm{dL})$

- Comparação: utilização de estatinas para atingir valores superiores ou não uso de estatinas

- Resultados: morbilidade CV e global (redução de eventos), mortalidade CV e global e efeitos laterais da terapêutica com estatina

Para avaliar a qualidade dos estudos e a força de recomendação foi utilizada a escala de Strength of Recommendation Taxonomy (SORT), da American Academy Family Physicians.

\section{RESULTADOS}

Na pesquisa inicial, 174 artigos foram identificados, cuja seleção se encontra explanada na Figura 1. Os resultados desta análise são apresentados no Quadro I em forma de síntese.

Na meta análise de Cholesterol Treatment Trialists' (CTT) Collaborators registou-se uma redução significativa da incidência de eventos vasculares major por $\mathrm{mmol} / \mathrm{L}$ de redução do c-LDL nos participantes com diabetes que foi semelhante à redução observada nas pessoas sem diabetes $(0,79$; IC99\% $0,76-0,82 ; p<0,0001)$. Entre os participantes com diabetes, esta redução foi semelhante, independentemente de história prévia de doença vascular (i.e., coronária, cerebrovascular ou arterial periférica), sexo, idade, hipertensão tratada, índice de massa corporal, história de tabagismo e taxa de filtração glomerular estimada, bem como do perfil lipídico inicial. Os autores constataram também que o benefício verificado foi independente dos valores de c-LDL pré-tratamento, pelo menos até valores de $2,6 \mathrm{mmol} / \mathrm{L}(\approx 100 \mathrm{mg} / \mathrm{dL})$, correspondentes a um valor de $2 \mathrm{mmol} / 1(\approx 77 \mathrm{mg} / \mathrm{dL})$ após estatina. ${ }^{8}$

Embora a maioria dos participantes dos estudos incluídos apresentasse DM tipo 2, não houve evidência 


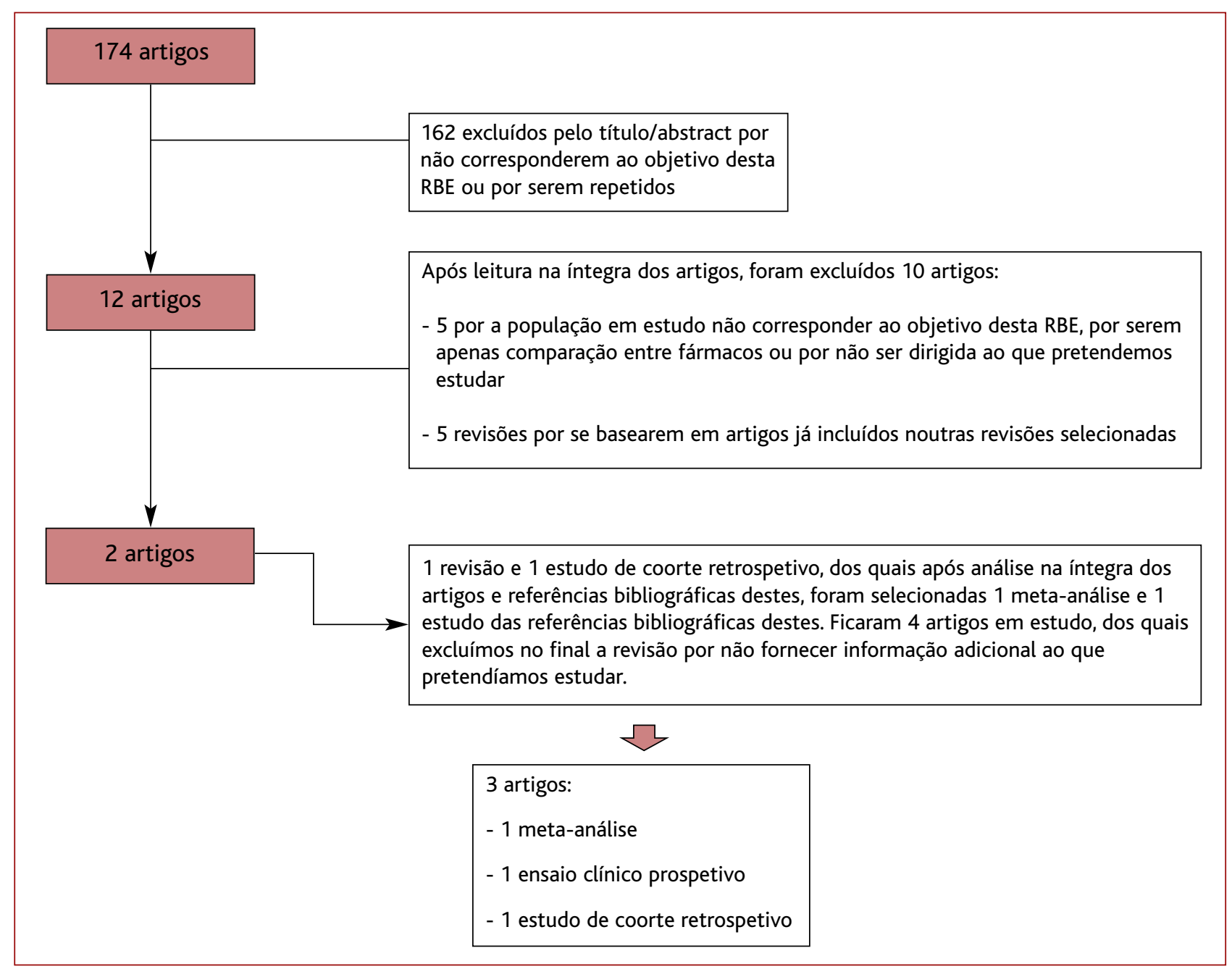

Figura 1. Organograma de seleção dos artigos para análise.

Legenda: RBE = Revisão baseada na evidência.

de que os efeitos da terapêutica com estatina na ocorrência de eventos vasculares major em pessoas com DM tipo 1 fossem diferentes daqueles com DM tipo 2 . A redução de eventos em pessoas com diabetes tipo 1 foi estatisticamente significativa, segundo os autores desta meta-análise. ${ }^{8}$

Verificaram-se também reduções significativas quando avaliados os eventos coronários major, revascularização coronária e acidente vascular cerebral (AVC), independentemente de terem ou não diabetes. ${ }^{8}$

O grupo de participantes com diabetes apresentou uma redução de $9 \%$ na mortalidade por todas as causas por mmol/L de redução de c-LDL (0,91, IC99\% 0,82-
$-1,01 ; p=0,02)$, que foi semelhante ao grupo sem diabetes, com uma redução de $13 \%(0,87$, IC99\% 0,82-0,92; $p<0,0001){ }^{8}$

Uma maior redução no valor do c-LDL foi associada a uma maior redução de eventos vasculares major, parecendo haver uma relação linear, sem existência de nenhum valor abaixo do qual este benefício não estivesse presente. $^{8}$

Como os 14 estudos acerca do efeito da terapêutica com estatina apresentaram resultados clínicos fatais e não fatais semelhantes entre os participantes com ou sem diabetes, os autores afirmam que a relação custo-eficácia do tratamento para uma pessoa, indepen- 


\begin{tabular}{|c|c|c|c|}
\hline Referência & População & Intervenção & Outcome \\
\hline $\begin{array}{l}\text { Cholesterol } \\
\text { Treatment } \\
\text { Trialists' } \\
\text { (CTT) } \\
\text { Collaborators } \\
(2008)^{8}\end{array}$ & $\begin{array}{l}14 \text { EC aleatorizados } \\
\text { (1994-2004): } \\
\text { - } 4 \text { S } \\
\text { - WOSCOPS } \\
\text { - CARE } \\
\text { - Post-CABG } \\
\text { - AFCAPS/TexCAPS } \\
\text { - LIPID } \\
\text { - GISSI-P } \\
\text { - LIPS } \\
\text { - HPS } \\
\text { - PROSPER } \\
\text { - ALLHAT-LLT } \\
\text { - ASCOT-LLA } \\
\text { - ALERT } \\
\text { - CARDS } \\
n=18.686 \text { pessoas com diabetes } \\
+71.370 \text { sem diabetes }\end{array}$ & $\begin{array}{l}\text { Terapêutica com estatina } \\
\text { Tempo médio de } \\
\text { seguimento: } 4,3 \text { anos }\end{array}$ & $\begin{array}{l}\text { Efeitos nos outcomes clínicos } \\
\text { (ocorrência de eventos vasculares } \\
\text { major, eventos coronários major, } \\
\text { necessidade de revascularização } \\
\text { coronária, AVC e mortalidade por } \\
\text { todas as causas) por redução de } \\
\approx 40 \mathrm{mg} / \mathrm{dL} \text { de redução no C-LDL }\end{array}$ \\
\hline $\begin{array}{l}\text { Prakash } \\
\text { Deedwania, } \\
\text { et al. }(2006)^{9}\end{array}$ & $\begin{array}{l}n=1.231 \text { pessoas com diabetes } \\
\text { Incluídos homens e mulheres } \\
\text { com idade entre } 35 \text { e } 75 \text { anos } \\
\text { com doença coronária } \\
\text { clinicamente evidente e com } \\
\text { síndroma metabólica } \\
\text { Subanálise do estudo Treating to } \\
\text { New Targets: } \\
\text { - prospetivo, } \\
\text { - duplo-cego } \\
\text { - de grupos paralelos } \\
\text { - realizado em } 256 \text { locais em } \\
14 \text { países, de abril de } 1998 \\
\text { a agosto de } 2004\end{array}$ & $\begin{array}{l}\text { Atorvastatina } 10 \mathrm{mg} \\
\text { ( } n=629) \text { vs } \\
\text { Atorvastatina } 80 \mathrm{mg} \\
(n=602) \\
\text { Tempo médio de } \\
\text { seguimento: } 4,9 \text { anos }\end{array}$ & $\begin{array}{l}\text { Outcome primário: } \\
\text { Tempo até à ocorrência de um } \\
\text { evento CV major: } \\
\text { - morte por doença cardíaca } \\
\text { coronária } \\
\text { - enfarte do miocárdio não fatal } \\
\text { não relacionado ao } \\
\text { procedimento } \\
\text { - paragem cardíaca com } \\
\text { ressuscitação } \\
\text { - AVC fatal ou não fatal } \\
\text { Outcomes secundários: } \\
\text { - evento CV } \\
\text { - evento coronário major } \\
\text { - evento coronário } \\
\text { - evento cerebrovascular } \\
\text { - doença arterial periférica } \\
\text { - insuficiência cardíaca } \\
\text { - congestiva com hospitalização } \\
\text { mortalidade por todas as causas }\end{array}$ \\
\hline
\end{tabular}

Legenda: MA = Meta-análise; EC = Ensaio clínico; $\mathrm{n}$ = Número amostral; c-LDL Colesterol LDL; NNT = Número necessário para tratar; AVC = Acidente vascular cerebral; $C V$ = Cardiovascular; Nível de evidência, segundo escala SORT. 
Nível de evidência

Redução com segurança da incidência de eventos vasculares major em cerca de $21 \%$ por $\approx 40 \mathrm{mg} / \mathrm{dL}$ de redução de c-LDL

(0,79; IC99\% 0,72-0,86; $p<0,0001)$ :

- eventos coronários major (0,78; IC99\% $0,69-0,87 ; p<0,0001)$

- revascularização coronária $(0,75$; IC99\% 0,64-0,88; $p<0,0001)$

- $\operatorname{AVC}(0,79 ;$ IC99\% 0,67-0,93; $p=0,0002)$ Redução de $9 \%$ na mortalidade por todas as causas por $\approx 40 \mathrm{mg} / \mathrm{dL}$ de redução de c-LDL

Reduções maiores no c-LDL foram associadas a maiores reduções proporcionais

Redução relativa de $\mathbf{2 9 \%}$ no risco de eventos
CV major em favor do grupo com dose alta

$(0,71 ;$ IC95\% 0,61-0,84; $p<0,0001)$

Redução de:

- qualquer evento CV $(0,78 ; 0,71-0,85$; $p<0,0001$ )

- evento coronário major $(0,72 ; 0,60-0,86$; $p=0,0004$ )

- qualquer evento coronário $(0,75 ; 0,67-0,83$; $p<0,0001)$,

- evento cerebrovascular $(0,74 ; 0,59-0,93$; $p=0,011$ )

- hospitalização por insuficiência cardíaca congestiva $(0,73 ; 0,55-0,96 ; p=0,027)$

Não houve diferença significativa para mortalidade por todas as causas dentemente da presença da diabetes, será a mesma. E como a dimensão do benefício depende principalmente da redução do c-LDL que é alcançada, poderá ser necessário rever as diretrizes atuais para garantir uma terapêutica com estatina suficiente para produzir uma redução substancial do c-LDL a todas as pessoas com diabetes, independentemente de terem desenvolvido doença vascular e do seu perfil lipídico. ${ }^{8}$

O estudo de Prakash Deedwania e colaboradores consiste numa subanálise do estudo TREATING TO NeW TARGETS (TNT), que foi um estudo prospetivo, duplamente-cego, de grupos paralelos realizado em 14 países (Austrália, Áustria, Bélgica, Canadá, França, Alemanha, Irlanda, Itália, Holanda, África do Sul, Espanha, Suíça, Reino Unido e Estados Unidos da América). Os pacientes elegíveis para inclusão foram homens e mulheres com idades entre 35 e 75 anos, com doença coronária clinicamente evidente, definida como enfarte do miocárdio prévio, angina prévia ou presente, com evidência objetiva de doença cardíaca coronária aterosclerótica ou procedimento prévio de revascularização coronária. Nesta subanálise foram incluídos aqueles que preenchessem os critérios para a síndroma metabólica (presença de três ou mais dos seguintes fatores de risco: $\mathrm{IMC} \geq 28 \mathrm{Kg} / \mathrm{m}^{2}$, triglicerídeos $\geq 150 \mathrm{mg} / \mathrm{dL}$, c-HDL $<40 \mathrm{mg} / \mathrm{dL}$ em homens e c-HDL $<50 \mathrm{mg} / \mathrm{dL}$ em mulheres, pressão arterial $\geq 130 / 85 \mathrm{mmHg}$ ou glicemia $\geq 100 \mathrm{mg} / \mathrm{dL}$ ). ${ }^{9}$

Os pacientes com síndroma metabólica, com e sem diabetes, tiveram um aumento progressivo no risco de eventos CV major à medida que o número de componentes da síndroma metabólica aumentou. Este achado sugere que aqueles com maior risco mostrarão um maior benefício com o incremento da dose de atorvastatina para 80mg ao longo de cinco anos. ${ }^{9}$

Em comparação com o estudo THE SCANDINAVIAN SimVASTATIN SuRvival Study (4S) (que comparava toma de sinvastatina $20 \mathrm{mg}$ ou $40 \mathrm{mg} v$ s placebo em doentes com doença coronária estável), em que a terapêutica com sinvastatina reduziu o risco de vários eventos de doença coronária em 30-40\% em comparação com o placebo, neste estudo a terapêutica com altas doses de atorvastatina reduziu ainda mais o c-LDL para $73 \mathrm{mg} / \mathrm{dL}$, indicando benefício clínico ainda mais significativo. Assim, esses achados apoiam a ideia de que uma maior redução do c-LDL que a obtida com doses padrão de 


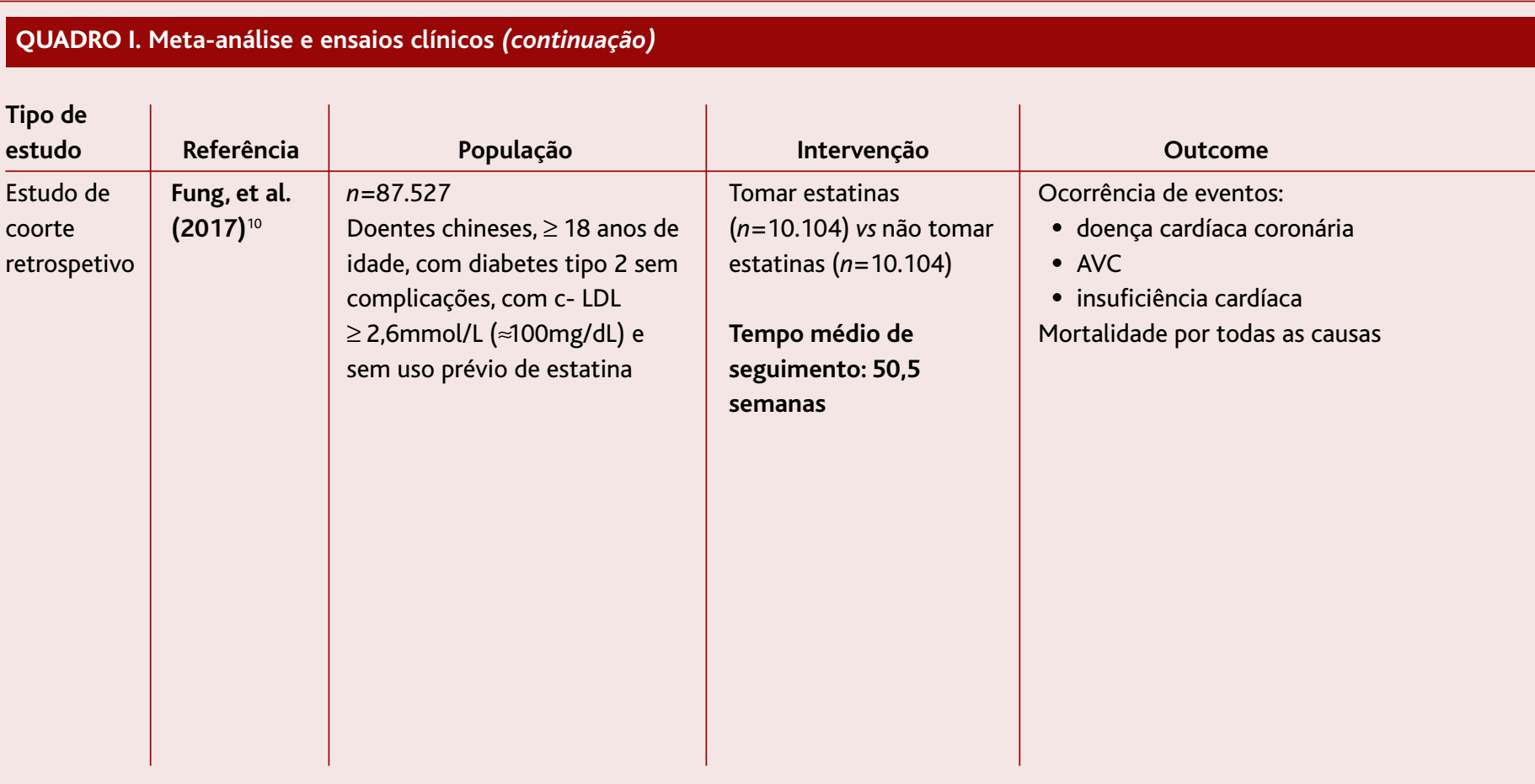

estatinas é justificada em pacientes com diabetes com doença coronária e síndroma metabólica. ${ }^{9}$

Apesar do alto número de pacientes com síndroma metabólica e da natureza robusta do desenho do TNT existem algumas limitações na aplicação desses resultados. Nomeadamente, o facto de este estudo ter envolvido apenas pacientes com doença cardíaca coronária clinicamente evidente, sendo que não podem ser extrapolados benefícios adicionais da terapêutica para obter valores além do objetivo atual de c-LDL de $2,6 \mathrm{mmol} / \mathrm{L}$ (100mg/dL) para pacientes com síndroma metabólica sem doença coronária. ${ }^{9}$ Nem é possível extrapolar para pacientes com diabetes sem síndroma metabólica. A definição de síndroma metabólica também não é universal, constituindo outra limitação neste estudo. ${ }^{9}$

O estudo de Fung e colaboradores incluiu utentes seguidos nos cuidados de saúde primários no ano de 2010 e sem uso anterior de estatinas. O estudo comparou inicialmente o grupo que iniciou estatinas $(n=14.132)$ e o grupo sem estatinas ( $n=73.395)$. Foram combinados com sucesso 10.104 indivíduos de cada grupo entre si em relação a todas as características da linha de base..$^{10}$
A subdivisão dos eventos CV em doença cardíaca coronária, AVC e insuficiência cardíaca também mostrou resultados consistentes com o da mortalidade por todas as causas e doença CV. ${ }^{10}$

Porém, o período de seguimento de cinco anos pode não ser suficiente para que algumas das complicações $\mathrm{CV}$ ou mesmo casos de mortalidade se desenvolvam; pelo que os autores referem que um período mais longo de acompanhamento poderia ajudar a confirmar se o uso prolongado de estatina ainda está associado a menor risco CV ou mortalidade por todas as causas. Várias limitações foram detetadas neste estudo, como a indisponibilidade de detalhes sobre o uso de estatina (incluindo a dose exata da estatina feita por cada paciente), a impossibilidade de avaliar a adesão à terapêutica com estatina prescrita e o não registo das modificações no estilo de vida dos pacientes (não sendo considerado o seu efeito na melhoria do perfil lipídico e na subsequente redução do risco CV). ${ }^{10}$

Relativamente a efeitos adversos da terapêutica com estatina para obter valores baixos de c-LDL, na meta-análise de Cholesterol Treatment Trialists' (CTT) Collaborators a terapêutica com estatinas não aumentou 
Nível de evidência

- Taxa de incidência de doença CV no grupo 2 com estatina de 16,533 por 1.000 pessoas-ano, enquanto o grupo de comparação teve 32,387 por 1.000 pessoas-ano: $\mathbf{N N T}=54$

- Taxa de incidência de 8,138 mortes por 1.000 pessoas-ano, enquanto o grupo de comparação teve 19,603 mortes por 1.000 pessoas-ano: $\mathbf{N N T}=\mathbf{5 1}$

No grupo a tomar estatina verificou-se redução de risco $C V$ em $\approx 50 \%$ e redução da mortalidade por todas as causas em $62,2 \%(p<0,001)$

No subgrupo em que alcançaram c-LDL $<2,6 \mathrm{mmol} / \mathrm{L}(\approx 100 \mathrm{mg} / \mathrm{dL})$ a redução do risco $\mathrm{CV}$ foi ainda maior portadas em $0,2 \%$ dos pacientes recebendo atorvastatina $10 \mathrm{mg}$ e $1,1 \%$ dos que recebem atorvastatina $80 \mathrm{mg}$. Nenhum dos pacientes, em ambos os grupos, teve elevações persistentes nas concentrações de creatinofosfoquinase. ${ }^{9}$

Por último, o estudo de Fung e colaboradores não faz referência a efeitos adversos associados à terapêutica com estatinas. ${ }^{10}$

\section{CONCLUSÕES}

Várias foram as limitações encontradas nos estudos analisados. Os estudos incluídos apresentam heterogeneidade na população em estudo, muitas vezes não fazendo distinção entre pessoas com diabetes com ou sem outros fatores de risco associados (alto risco $v s$ muito alto risco) e um dos estudos é relativo unicamente a doentes em prevenção secundária, sendo difícil extrapolar os resultados destes estudos de acordo com as recomendações atualmente existentes. Além disso, não foi possível em muitos destes estudos confirmar a adesão a determinada terapêutica ou mesmo mudanças do estilo de vida possivelmente implementadas e que possam interferir com os resultados obtidos.

Outras limitações são o facto dos estudos incluídos não terem como objetivo a análise de possíveis efeitos adversos da terapêutica com estatina (registando-se num deles ausência à sua referência) e do período de seguimento nestes estudos ter sido de aproximadamente quatro a cinco anos, período que pode não ser suficiente para avaliar possível impacto desta terapêutica intensiva na mortalidade por todas as causas, afetando a avaliação do risco-benefício desta abordagem a longo prazo.

De ressalvar, por fim, que nenhum dos estudos foi realizado na população portuguesa, o que pode condicionar a interpretação das conclusões por refletirem uma realidade distinta, e que alguns destes estudos foram patrocinados pela indústria farmacêutica, aumentando também a preocupação sobre potenciais vieses.

Relativamente aos valores alvo de c-LDL adequados existe muito pouca evidência atualmente disponível, referindo-se muitas vezes a uma redução absoluta de c-LDL associada a benefício proporcional na morbilidade e mortalidade e não a uma redução para atingir 
um determinado valor de c-LDL, nem a titulação de dose de estatinas (como apresentado em algumas das normas americanas disponíveis na atualidade). Nos estudos apresentados existe evidência consistente de que a redução de c-LDL produz benefícios na morbilidade (ocorrência de eventos) proporcionais a essa redução; no entanto, na melhoria da mortalidade por todas as causas, a evidência torna-se inconsistente, não existindo comprovadamente uma proporcionalidade entre a redução do c-LDL e os seus benefícios sobre a mortalidade.

Concluindo, parece haver benefício da terapêutica com estatinas para obter valores baixos de c-LDL na redução de morbilidade e mortalidade (força de recomendação B) nos utentes com diabetes. Contudo, existe evidência limitada sobre os valores alvo de c-LDL recomendados, nomeadamente para o valor alvo especificamente abaixo de 70mg/dL. Assim, apesar destes resultados serem encorajadores, os médicos devem discutir com os pacientes o seu plano de abordagem a longo prazo, com uma avaliação cuidadosa e individualizada dos riscos e benefícios que possam decorrer desta terapêutica intensiva; nunca esquecendo também o papel importante das modificações no estilo de vida, incluindo dieta saudável, exercício físico regular e controlo de peso, e oferecendo uma abordagem individualizada a cada pessoa com diabetes, dadas as particularidades desta população em estudo.

São, deste modo, necessários mais estudos, nomeadamente ensaios controlados, aleatorizados e de qualidade, com maior tempo de seguimento, que permitam a evidência do real benefício na redução intensiva de c-LDL na morbilidade e mortalidade nas pessoas com diabetes, bem como dos respetivos valores alvos de c-LDL a atingir.

\section{REFERÊNCIAS BIBLIOGRÁFICAS}

1. Enas EA, Kuruvila A, Khanna P, Pitchumoni CS, Mohan V. Benefits \& risks of statin therapy for primary prevention of cardiovascular disease in
Asian Indians: a population with the highest risk of premature coronary artery disease \& diabetes. Indian J Med Res. 2013;138(4):461-91.

2. Taylor F, Huffman MD, Macedo AF, Moore TH, Burke M, Smith GD, et al. Statins for the primary prevention of cardiovascular disease. Cochrane Database Syst Rev. 2013;(1):CD004816.

3. Direção-Geral da Saúde. Abordagem terapêutica das dislipidemias no adulto: norma n. ${ }^{\circ}$ 019/2011, de 28/09/2011, atualizada em 11/05/2017. Lisboa: DGS; 2017.

4. Direção-Geral da Saúde. Avaliação do risco cardiovascular SCORE (Systematic Coronary Risk Evaluation): norma n. ${ }^{\circ} 005 / 2013$, de 19/03/2013, atualizada em 21/01/2015. Lisboa: DGS; 2015.

5. Catapano AL, Graham I, De Backer G, Wiklund O, Chapman MJ, Drexel $\mathrm{H}$, et al. 2016 ESC/EAS Guidelines for the management of dyslipidaemias. Eur Heart J. 2016;37(39):2999-3058.

6. Stone NJ, Robinson JG, Lichtenstein AH, Merz CN, Blum CB, Eckel RH, et al. 2013 ACC/AHA Guideline on the treatment of blood cholesterol to reduce atherosclerotic cardiovascular risk in adults: a report of the American College of Cardiology/American Heart Association Task Force on Practice Guidelines. J Am Coll Cardiol. 2014;63(25 Pt B):2889-934.

7. American Diabetes Association. Cardiovascular disease and risk management: standards of nedical care in diabetes - 2018. Diabetes Care. 2018;41(Suppl 1):S86-S104.

8. Cholesterol Treatment Trialists' (CTT) Collaborators. Efficacy of cholesterol-lowering therapy in 18686 people with diabetes in 14 randomised trials of statins: a meta-analysis. Lancet. 2008;371(9607):117-25.

9. Deedwania P, Barter P, Carmena R, Fruchart JC, Grundy SM, Haffner S, et al. Reduction of low-density lipoprotein cholesterol in patients with coronary heart disease and metabolic syndrome: analysis of the treating to new targets study. Lancet. 2006;368(9539):919-28.

10. Fung CS, Wan EY, Chan AK, Lam CL. Statin use reduces cardiovascular events and all-cause mortality amongst Chinese patients with type 2 diabetes mellitus: a 5-year cohort study. BMC Cardiovasc Disord. 2017;17(1):166.

\section{CONFLITO DE INTERESSES}

Os autores declaram não ter quaisquer conflitos de interesse.

\section{ENDEREÇO PARA CORRESPONDÊNCIA}

Carla Longras

E-mail: carlalongras@hotmail.com

https://orcid.org/0000-0002-6816-471X

Recebido em 25-02-2019

Aceite para publicação em 05-06-2019 


\section{ABSTRACT \\ THE IMPACT OF INTENSIVE LDL-C REDUCTION ON MORBIDITY AND MORTALITY IN PATIENTS WITH DIABETES: AN EVIDENCE-BASED REVIEW}

Objective: The aim of this review is to determine the impact of LDL-c reduction with statins on morbidity and mortality in patients with diabetes

Methods: The search included studies, published between $1^{\text {st }}$ January 2008 and $19^{\text {th }}$ July 2018, in English, with the MeSH terms: Hydroxymethylglutaryl-CoA Reductase Inhibitors AND diabetes mellitus AND low-density lipoprotein AND morbidity; Hydroxymethylglutaryl-COA Reductase Inhibitors AND diabetes mellitus AND low-density lipoprotein AND mortality. To assign the levels of evidence and recommendation forces, the American Academy of Family Physicians (AAFP) SORT scale was used.

Results: A total of 174 articles were identified and three were selected: one meta-analysis and two clinical trials. The meta-analysis included 14 studies and demonstrated the benefit of statins in reducing the incidence of major vascular events in about $1 / 5$ per $\mathrm{mmol} / \mathrm{L}$ of $\mathrm{LDL}-\mathrm{c}$ reduction. In the prospective clinical trial, the reduction of events was greater in the group with greater LDL-c reductions but there were no differences in all-cause mortality. In the retrospective clinical trial, there was a reduction of cardiovascular risk and all-cause mortality in the group assigned to statins compared to the control group.

Conclusions: There were several limitations in the included studies, namely heterogeneous populations, short follow-up and methodological shortcomings. There seems to be a benefit in taking statins to lower LDL-c levels on the reduction of morbidity, cardiovascular events and all-cause mortality (strength of recommendation B). However, there is limited evidence about the recommended target LDL-c levels.

Keywords: Hydroxymethylglutaryl-CoA reductase inhibitors; Diabetes mellitus; Low-density lipoprotein; Morbidity; Mortality. 